\title{
Epidemiologic, Clinical and Radiological Aspects of Male Urethral Strictures at the Ngaoundéré Regional Hospital
}

\author{
Mathurin Neossi Guena ${ }^{1,2 *}$, Florent Zilbinkai Alapha ${ }^{2}$, Eric Lonang Wandji, \\ Odile Fernande Zeh ${ }^{3,4}$, Joseph Gonsu Fotsing,4 \\ ${ }^{1}$ Department of Biomedical Sciences, Faculty of Sciences, University of Ngaoundéré, Ngaoundéré, Cameroon \\ ${ }^{2}$ Imaging Service, Ngaoundéré Regional Hospital, Ngaoundéré, Cameroon \\ ${ }^{3}$ Faculty of Medicine and Biomedical Sciences, University of Yaounde I, Yaounde, Cameroon \\ ${ }^{4}$ Yaounde Gynaecology Obstetrics and Pediatrics Hospital, Yaounde, Cameroon \\ Email: *mneossiguena@yahoo.fr
}

How to cite this paper: Guena, M.N., Alapha, F.Z., Wandji, E.L., Zeh, O.F. and Fotsing, J.G. (2019) Epidemiologic, Clinical and Radiological Aspects of Male Urethral Strictures at the Ngaoundéré Regional Hospital. Open Journal of Radiology, 9, 115-124.

https://doi.org/10.4236/ojrad.2019.92011

Received: April 27, 2019

Accepted: June 1, 2019

Published: June 4, 2019

Copyright $\odot 2019$ by author(s) and Scientific Research Publishing Inc. This work is licensed under the Creative Commons Attribution International License (CC BY 4.0).

http://creativecommons.org/licenses/by/4.0/

\begin{abstract}
Background: Urethral stricture is a disabling condition that remains prevalent due to the upsurge of sexually transmitted infections and traumatic lesions of the urethra during trauma of the pelvis in humans. Objective: Describe the epidemiological, clinical and radiological presentations of male urethral strictures. Method: It was a cross-sectional and descriptive study during a one-year period from January to December 2017 including all male patients presenting for a urethrocystography examination, at the Ngaoundere Radiology and Medical Imaging Center who agreed to participate. The urethrocystography technique depended on patient's condition and was either retrograde, anterograde, or following intravenous urography. The examinations were performed with GE X-ray equipment using Kodak Dry View CRs. The interpretation was performed by a radiologist with at least five years of experience. The variables studied were age, clinic, history, uretrocystography technique and results. The data were collected using a previously established data sheet and processed with Sphinx Plus2 V5 and Microsoft Excel 2010 software. Results: 39 patients were enrolled in this study; the average age of our patients was 43.8 years with extremes of 4 and 76 years. The $50-60$ age group (30.7\%) was the most affected. Infection (53.9\%) and trauma (23.1\%) were the most common etiologies; dysuria (38.5\%) and diminution of urinary flow $(30.8 \%)$ were the most patient's complaint. Retrograde urethrocystography (66.7\%) was the most performed technic. Minimal bleeding in $46.2 \%$ of patients was the most common incident during urethrocystography; $76.9 \%$ of patients had a difficult urination and $7.7 \%$ an impassable urethral meatus. After interpretation, $84.6 \%$ of patients had urethral stenosis, which
\end{abstract}


was associated with bladder diverticula (23\%) and fistulas (15.3\%). The stenosis was seated on membranous urethra (81.8\%), tight type (45.4\%), short $(66.7 \%)$ and single $(81.8 \%)$. The post-void residue was significant in $84.6 \%$ of cases and not significant in $15.4 \%$ of cases. $15.3 \%$ of the urethrocystographies were normal. Conclusion: Urethral stenosis in Ngaoundere mainly affects young adult; the main etiology is infectious, very often unique, short, and tight and the membranous urethra is the seat of predilection. Hence, to reduce the extent of pathology with its functional consequences and its impact on the socio-professional life in our context, the focus should be on sexually transmitted infectious.

\section{Keywords}

Stenosis, Urethrocystography, Male Urethra

\section{Introduction}

Stenosis of the urethra can be defined as a reduction in the size, more or less extended, of the urethral canal. It is a relatively common condition among men with a prevalence of 229 - 627 per 100,000 inhabitants or $0.6 \%$ of the population at risk; it affects men of all ages with a peak prevalence between 40 and 45 years and is very rare under 9 years [1] [2] [3]. Its incidence is currently increasing gradually because of the increased number of permanent catheter carriers, the increase in sexually transmitted diseases (STDs) and the misuse of transurethral diagnostic or therapeutic instruments [3]. In Africa, and especially in the tropics, the frequency is not negligible, according to Ouattara K et al. [4]. Although infectious forms are decreasing in developed countries in favor of post-traumatic and iatrogenic forms, this does not seem to be the case in our context [3]. In patients with urethral stenosis, patients experience a weak urinary flow, difficulty urinating, incomplete emptying, urinary retention and recurrent urinary tract infections [1]. The evolution of urethral stenosis will lead to a more or less complete obstruction of the lower urinary tract with, in the long run, an impairment of renal function and a risk of chronic renal failure. Despite the progress made, care is still problematic in many situations [5]. Radiology is the basis for the diagnosis of urethral strictures; conventional imaging of the urethra with urethrocystography is easy to perform, available, reproducible and economical examination that can detect clinically relevant narrowing involving the urethra [3], making it the first choice imaging for suspicion of stenosis in developing countries. Radiographic patterns of strictures, which included the number, site, types, length and associated radiographic findings as well as complications, were obtained from the contrast urethrograms. In addition, it is a pathology of the adult in professional activity, provider of psychological disorders and disabling, which can generate significant medical, socio-economic costs and a negative impact on the quality of life of patients. Understanding the epidemiology, clinical and radi- 
ological pattern of urethral strictures can identify risk factors for the onset or progression of the disease, which can be the subject of preventive measures to reduce the severity of the disease and health expenses, so the purpose of this work was to identify the etiological, sociodemographic, clinical and radiological features of urethral stenosis in the Ngaoundere Regional Hospital.

\section{Method}

It was a cross-sectional and descriptive study that took place over one year from January to December 2017 at the radiology and medical imaging center of the Ngaoundéré Regional Hospital. All male patients referred for an urethrocystography examination, and having deliberately agreed to participate was included in our study. The examinations were performed with GE bone-Lung X-ray equipment using the Kodak Dry View CR. The examination was done by appointment in order to better prepare the patient, among other things to ensure the absence of contrast medium the days preceding the examination, to look for the history of allergy to consider a possible premedication, evacuation if necessary of the feces in the rectal bulb and the sigmoid by an evacuating enema. The technique used was either retrograde, anterograde urethrocystography or following intravenous urography depending on the patient's condition. The examination starts with the taking of $\mathrm{x}$-ray picture without injection of contrast medium, during injection of contrast medium, during urination and after urination, all in strict respect of the asepsis. The interpretation was performed by a radiologist with at least five years of experience. The variables studied were age, clinic, antecedents, technic and results of urethrocystography. The data was collected using a previously established data sheet and processed with Sphinx Plus2 V5 and Microsoft Excel 2010 software.

\section{Results}

\subsection{Epidemiological and Clinical Characteristics}

The study included 39 patients aged 4 to 76 years, with an average of 43.8 years. The age groups 50 - 60 years (31\%), $30-40(23.07 \%)$ years and over 60 years (23.07\%) were the most affected (Figure 1). Shepherds (23\%), drivers (23\%), administrators (15.3\%), soldiers (15.3\%) and students (15.3\%) were the most represented socio-professional categories.

Dysuria (69.23\%), urinary flow reduction (15.4\%) and droplet urination (15.4\%) were the most common clinical manifestations (Table 1). The etiologies observed were infection (53.9\%), trauma (23.1\%), iatrogenic (8\%), in $15 \%$ of cases the etiology was not known (Figure 2).

Urologists (54\%), surgeons (38\%) and general practitioners (8\%) were the main prescribers for urethrocystography.

\subsection{Radiological Aspects}

Urethrocystography (UCG) performed was retrograde (61.53\%), following 


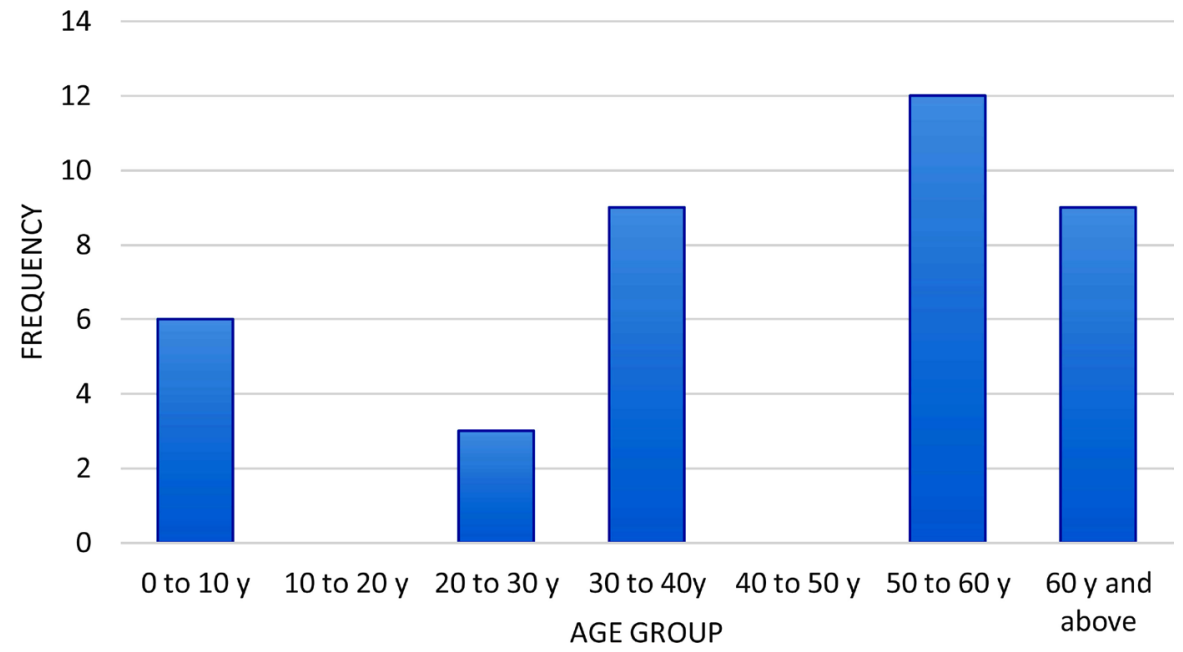

Figure 1. Distribution of patients by age group.

Table 1. Distribution of indications.

\begin{tabular}{ccc}
\hline Patient's complaint & Frequency & Percentage (\%) \\
Dysuria & 27 & 69.23 \\
Urinary flow reduction & 6 & 15.4 \\
Droplet urination & 6 & 15.4 \\
Urinary incontinence with intermittent \\
$\begin{array}{c}\text { urinary retention antecedent } \\
\text { Suspicion of urethral fistula } \\
\text { Urinary retention }\end{array}$ & 3 & 7.7 \\
Rupture of the urethra following road accident & 3 & 7.7 \\
\hline
\end{tabular}

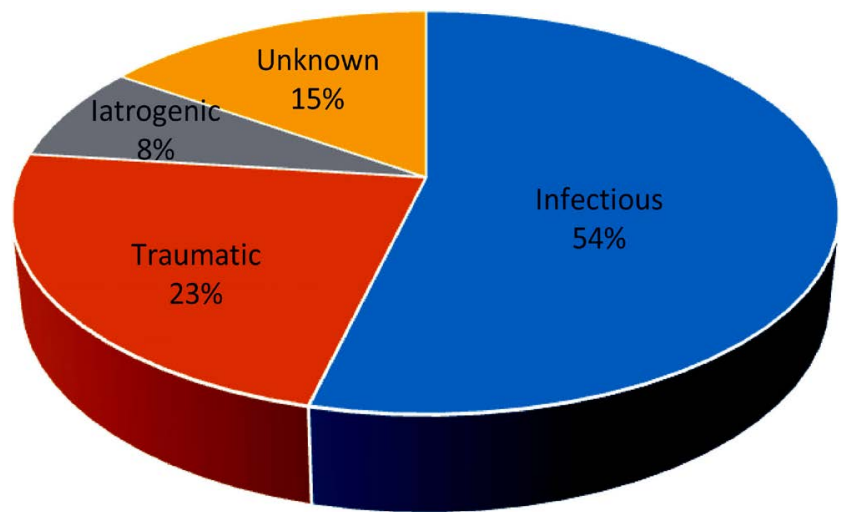

Figure 2. Urethral stricture etiology.

intravenous urography (15.38\%), retrograde and antegrade (15.38\%) and antegrade (7.7\%). The main difficulties encountered during urethrocystography were difficult urination in $20(76.9 \%)$ patients and an impassable urethral meatus in 2 (7.7\%) patients, the incidents observed during the examinations were essentially minimal bleeding (46.2\%). Stricture (84.61\%) accounted for most of the lesions observed, associated with bladder diverticula (23\%) and fistulas (15.3\%). 15.3\% 
of examinations were normal (Table 2).

Table 3 presents the radiological patterns of urethral stenosis, the most common location was the membranous urethra (81.1\%), followed by the bulbar (9\%) and penis urethra (9\%), the stenosis was short $(\leq 20 \mathrm{~mm})$ in $66.7 \%$ and long $(>20$ $\mathrm{mm}$ ) in $33.3 \%$, it was loosely tightened in $18.1 \%$, tight in $45.4 \%$ and complete in $36.3 \%$, single in $81.1 \%$ and multiple in $18.9 \%$.

Figure 3 is an oblique incidence of retrograde urethrocystography in a patient with dysuria for several months, showing a double stenosis respectively of the membranous urethra, which is tight and long, of the bulbar urethra, which is short and loosely tightened.

Figure 4 shows an oblique incidence of retrograde urethrocystography demonstrating a single stenosis of the bulbar urethra, which tight and long, associated with large diverticula of the bladder.

\section{Discussion}

\subsection{Sociodemographic and Clinical Characteristics}

The stenosis of the urethra reached all age categories ( 4 to 76 years) in our series

Table 2. Distribution of UCG results.

\begin{tabular}{ccc}
\hline UCG results & Frequency & Percentage (\%) \\
\hline Stricture & 18 & 46.1 \\
Stricture + diverticula & 9 & 23 \\
Stricture + fistula & 6 & 15.3 \\
Normal exam & 6 & 15.3 \\
Total & 39 & 100
\end{tabular}

Table 3. Radiological aspect of stricture.

\begin{tabular}{|c|c|c|c|}
\hline Radiological & aspects of stricture & Frequency & Percentage (\%) \\
\hline \multicolumn{4}{|l|}{ Seat of striture: } \\
\hline & - Penis urethra & 3 & 9 \\
\hline & - Bulbar & 3 & 9 \\
\hline & - Membranous & 27 & 81.1 \\
\hline \multicolumn{4}{|l|}{ Length: } \\
\hline & - $\leq 20 \mathrm{~mm}$ & 22 & 66.7 \\
\hline & - $>20 \mathrm{~mm}$ & 11 & 33.3 \\
\hline \multicolumn{4}{|l|}{ Type: } \\
\hline & - Little tight & 6 & 18.1 \\
\hline & - Tight & 15 & 45.4 \\
\hline & - Complete & 12 & 36.3 \\
\hline \multicolumn{4}{|l|}{ Number: } \\
\hline & - Single & 27 & 81.1 \\
\hline & - Multiple (three) & 6 & 18.1 \\
\hline
\end{tabular}




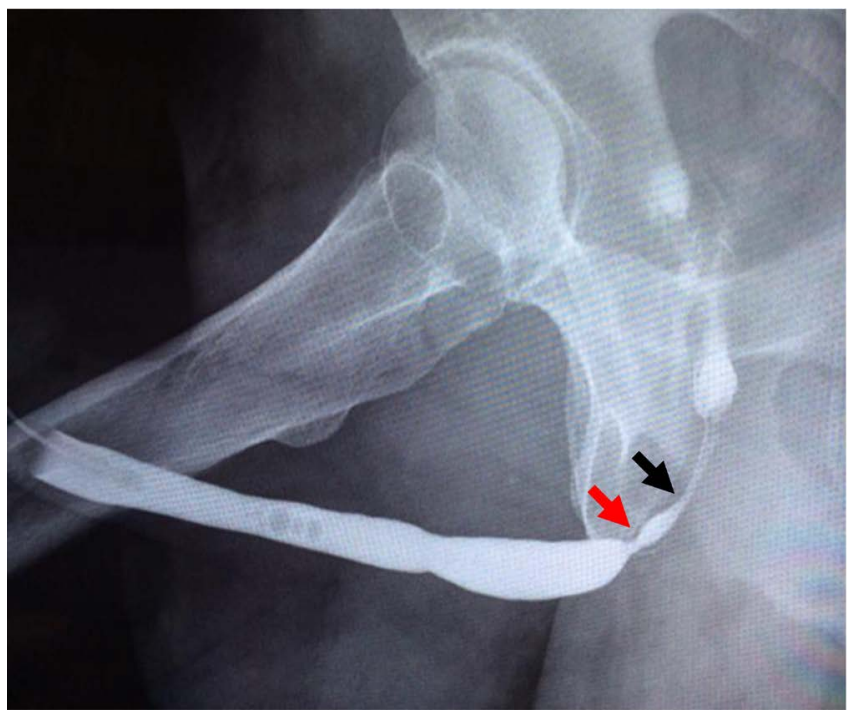

Figure 3. Membranous urethral (black arrow) and bulbar stricture (black arrow).

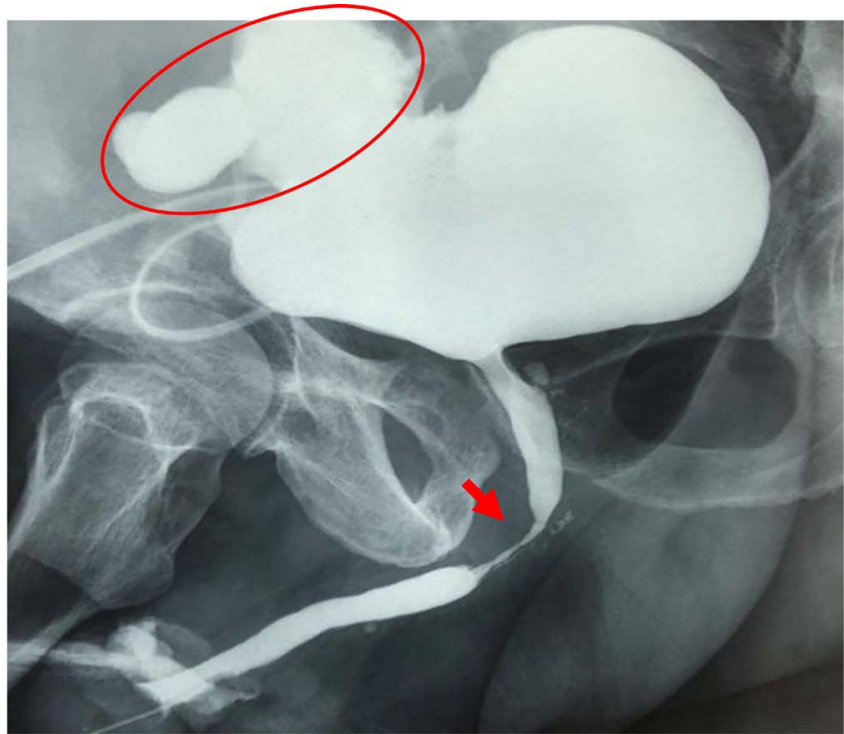

Figure 4. Bulbar urethral stricture (arrow) bladder diverticula (circle).

with an average age of 43.8 years, however the age group most concerned was that of 50 - 60 years. Some authors have found an average age of 40 years with extremes of 9 and 78 years, averages between 25 and 55 years and extremes of 0.06 and 90 years [5] [6] [7] [8]. All these studies showed that stenosis appeared at any age, but was mostly a disease of young adults between 30 and 50 years of age. Shepherds and drivers were the most represented profession in our series with a frequency of $23.1 \%$. This is explained by the precarious situation of shepherds and some drivers who by shame or lack of funds avoid urological consultation and prefer to use the care of traditional healers and sometimes unqualified health workers. This has a tendency to aggravate this pathology with a reduction in the size of the urethra, hence the presence of dysuria, which was the main reason for consultation found in our study, for about $69.23 \%$. This is a 
major telltale sign of urethral stricture which may be insidious [9]. It represented $55 \%, 58.6 \%$ and $88.9 \%$ in the respective studies of Djé et al. in Côte d'Ivoire, Diakhité and al, Ouattara et al. in Mali [9] [10] [11]. This is a difficulty in urination in connection with a decrease of the urethral canal, whose etiologies are variable. These etiologies are dominated by infections in our series (53.9\%) followed by trauma (23.1\%) but also in those of Ahmed and al, Djéet al. which reported $66.5 \%$ and $87 \%$ infectious respectively [9] [12]. According to Ngaroua and al, these results could be explained by the frequency of urogenital infections in tropical environments, but also the low socioeconomic level of the population, self-medication, multiple road accidents and late consultations [13]. In contrast to the southern countries, the etiology of urethral stenosis in developed countries has become iatrogenic due to the development of endoscopic maneuvers [10]. In our context the iatrogenic cause is poorly represented with about $8 \%$ and is due to repeated bladder catheterization, the insufficiency in the training of the personnel involved in the realization of these gestures increases the frequency of occurrence of the urethral stenosis [13] [14] [15]. About 15\% of the stenoses were of unknown origin, according to Seydou S. and Coulibaly these strictures would be stenoses of meatus, probably due to bullous affections affecting the glans or to sclerotrophic lichens [14]. The management of this pathology was mainly performed by urologists in $53.9 \%$ followed by general surgeons $38.5 \%$, In fact the urologist who is a specialist in urinary pathologies is at the forefront of the management of patients who are suspected of stenosis of the urethra.

\subsection{Radiological Aspects}

Retrograde urethrocystography (61.53\%) was the most common technic. Performed under satisfactory technical conditions, it gives excellent results highlighting the narrowing, allow to measure its extent, and especially to assess the importance of lesions of peri urethral spongy body, but however it has limitations due to the variation in position and traction of the penis that can greatly change the appearance of stenotic areas [16] [17]. Of the 39 urethrocystograms performed, $33(84.7 \%)$ had stenosis and $6(15.3 \%)$ were normal. This could be explained by the fact that the majority of our patients were consulted by the urologists who are the specialists of these diseases and who pose well the indication of these examinations. The analysis of the radiological features of strictures found in patients revealed that they were unique in $81.1 \%$ of cases, which is consistent with the results obtained in the Ndémanga Kamoune and Benjelloun series [5] [18]. On the other hand, Ahmed Ahidjo et al. and Ouattara reported a marked predominance of multiple forms in respective proportions of $85 \%$, $68.9 \%$ and $53.5 \%$ [10] [12] [19]. Urethral narrowing affects the different parts of the urethra [12]. In our study, the most common site was membranous, $81.1 \%$. These data are different from those found by the work of Ndemanga and al, Musau and al, for whom the preferred site is the urethral bulb, because of the 
predominant inflammatory etiology, the numerous para-urethral glands at the level of the bulb favor infection in this site and subsequent stenosis [5] [20]. In our series, these stenoses were tight (45.5\%) and very tight (36.3\%). KHLIL Sara in her study on post-traumatic stenosis had found that the stenosis of the urethra was complete in $60 \%$ of cases and partial in $40 \%$ of cases [21], the infectious etiology (53.9\%) was dominant in our context, which would explain the fact that stenoses are partial. The length of these strictures varied from 0.7 to $2 \mathrm{~cm}$ (66.7\%) and from 2.4 to $2.7 \mathrm{~cm}$ (33.3\%), which corroborates the results of Moukine Billah who had found a predominance of short stenosis (58.33\%) [22] and Ahmed Ahidjo et al. who observed in their study that stenosis lengths ranged from 2 to $25 \mathrm{~mm}$ in $74.3 \%$ of cases [12] [19]. These stenoses were associated with bladder diverticula (23\%) and fistulas (15.3\%), which corroborates the study of Ahmed Ahidjo et al. who reported as a pathological association to stenosis, diverticula, fistulas, stones and vesico ureteral reflux [19]. 84.6\% of our patients had a post-voiding residue that could have a longer-term impact on the upper urinary tract with a risk of obstructive renal failure, hence, the need for manual emptying of the bladder of patients with significant post-voiding residue.

Some limitations were however encountered during this study, including the fact that we have not studied the therapeutic aspects or even the follow-up of the patients; we did not evaluate the complications of this pathology especially on the upper urinary system.

\section{Conclusion}

The urethral stenosis in the male subject in Ngaoundere mainly affects the young adult, manifested clinically by dysuria and weakness of the urinary stream; its main etiology is infectious. Diagnosis arises only by urethrocystography; the membranous urethra is the site of choice, tight type, short and unique in most cases. The main complications are bladder diverticula and urethral fistulas. Focusing on the prevention of sexually transmitted infections would help to reduce the extent of this pathology in our context with its functional consequences and their impact on the socio-professional life.

\section{Conflicts of Interest}

The authors do not declare any conflict of interest.

\section{References}

[1] Alwaal, A., Blaschko, S.D., McAninch, J.W. and Breyer, B.N. (2014) Epidemiology of Urethral Strictures. Translational Andrology and Urology, 3, 209-213.

[2] Santucci, R.A., Joyce, G.F. and Wise, M. (2007) Male Urethral Stricture Disease. Journal of Urology, 177, 1667-1674. https://doi.org/10.1016/j.juro.2007.01.041

[3] El-Ammari, J.E., El-Yazami, O., El-Fassi, M. and Farih, M. (2012) Termino-Terminal Urethrorraphy the Treatment of Stricture of the Bulbar and Membranous Urethra. African Journal of Urology, 17, 66-71.

[4] Ouattara, K., Koungoulba, M.B. and Cisse, C. (1990) Realities of Narrowing of the 
Urethra in Tropical Zone. About Seventy-One Cases. Urology Annals, 24, 287-292.

[5] Kamoune, N., Doui Doumgba, A., Khaltan, E. and Mamadou, N.N. (2006) Stenosis of the Male Urethra in Bangui (CAR): Epidemiological Approach from 69 Files Collected at the Urology Department of the Hospital of the Friendship. Med Afr Noire, 53, 645-650.

[6] Dakum, N.K., Ramyil, V.M. and Amu, C.O. (2008) Outcome of Urethroplasty for Urethral Stricture at Jos University Teaching Hospital. Nigerian journal of clinical practice, 11, 300-304.

[7] Al-Ba'adani, T.H., Al-Asbahi, W., Al-Towaity, M., et al. (2010) Urethral Stricture Yemen Experience. International Urology and Nephrology, 42, 703-708. https://doi.org/10.1007/s11255-009-9657-3

[8] Tijani, K.H., Adesanya, A.A. and Ogo, C.N. (2009) The Newpattern of Urethral Stricture in Lagos. Nigerian Postgraduate Medical Journal, 16, 162-165.

[9] Djé, K., Coulibaly, N., Coulibaly, A. and Sangaré, I.S. (1999) Endoscopic Internal Urethrotomy in the Treatment of Acquired Urinary Stricture of African Black: About 140 Cases. Med Afr Noire, 46, 56-61.

[10] Ouattara, Z., Tembély, A., Sanogo, ZZ., Doumbia, D., Cissé, C.M.C. and Ouattara K. (2004) Narrowing of the Urethra in Men at the G Point Hospital. Le Mali médical, 19, 48-50.

[11] Diakhité ML., Fofana T., Sanogo, M., Kane, S.A., Ouattara, Z. and Ouattara, K. (2012) The Narrowing of the Urethra at CHU Gabriel Touré. About 77 Cases. Med Afr Noire, 59, 193-198.

[12] Ahmed, A. and Kalayi, G.D. (1998) Urethral Stricture at Ahmadu Bello University Teaching Hospital, Zaria. East African Medical Journal, 75, 582-585.

[13] Ngaroua, N.J.E., Djibrilla, Y., Asmaou, O. and Mbo, A.J. (2017) Epidemiological, Clinical and Management Aspects of Urethral Stenosis in Adults in a District Hospital in Ngaoundere, Cameroon. Pan African Medical Journal, 26, 193.

[14] Coulibaly, S. (2007) Therapeutic Aspects of Urethral Stenosis in Humans in the Urology Department of HUC GABRIEL TOURE. Medicine Thesis, Faculty of Medicine, Pharmacy and Odonto-Stomatology. Bamako, Mali.

[15] Ze Ondo, C., Fall, B., Diallo, Y., et al. (2015) The Iatrogenic Urethral Stricture: Experience of a Senegalese Hospital. African Journal of Urology, 21, 144-147. https://doi.org/10.1016/j.afju.2015.03.003

[16] Abou El-ghar, M., Osman, Y., Elbaz, E., Refiae, H. and El-Diasty, T. (2010) MR Urethrogram versus Combined Retrograde Urethrogram and Sonourethrography in Diagnosis of Urethral Stricture. European Journal of Radiology, 74, e193-e198. https://doi.org/10.1016/j.ejrad.2009.06.008

[17] Oosterlinck, W., Van Cauwenberghe, G. and Lumen, N. (2006) Endoscopic Treatment of Stenoses of the Urethra. Surgical Techniques. Annales d Urologie, 41, 322.

[18] Benjelloun, M., Drissi, M., Makhloufi, M., Karmouni, T., Tazi, K., Elkhader, K., et al. (2008) Treatment of Stenosis of the Urethra by Endoscopic Internal Urethrotomy: Anatomical and Functional Results of a Series of 244 Cases. African Journal of Urology, 14, 114-119. https://doi.org/10.1007/s12301-008-0002-3

[19] Ahidjo, A., Abdulkadir, A.Y., et al. (2012) Evaluation of Radiographic Pattern of Male Urethral Strictures in Nigeria: A Preliminary Report of a Proposed New Scoring System for Developing Countries. Iranian Journal of Radiology, 9, 60-62. https://doi.org/10.5812/iranjradiol.6357

[20] Musau, P. and Mteta, A. (2007) Uretrhal Strictures in a in a Tertiary Care Hospital 
in Tanzania. East African Medical Journal, 84, 522-524.

[21] Sara, K. (2013) Posttratraumatic Stenosis of the Membranous Urethra (About 15 cases). Thesis for Obtaining a Doctorate in medicine. Faculty of Medicine and Pharmacy FES, SIDI University Mohammed Ben Abdellah, Morocco.

[22] Billah, M.M. (2010) Termino-Terminal Urethrorrhaphy for Bulbar Urethral Stenosis (about 24 Cases). Medicine Thesis. Faculty of Medicine and Pharmacy, Mohammed V University, Rabat, Morocco. 